\title{
ECOFLORE, UN LOGICIEL SIMPLE DE DIAGNOSTIC ÉCOLOGIQUE
}

\author{
M. BARTOLI - M. TRAN-HA - G. LARGIER \\ G. DUMÉ - L. LARRIEU
}

Ecoflore est dédié à Jean-Claude Rameau dont nous avons, avec ses encouragements, utilisé le grand savoir.

La "valeur indicatrice des plantes" ou, en reflet, "les exigences de chaque espèce" sont la base de la phytoécologie. Dans la lignée d'ouvrages associant détermination des espèces et information sur leur autécologie, un des apports les plus novateurs de la récente Flore forestière française (1) (Rameau et al., 1989 et 1992) est de proposer une structuration de la végétation en groupes d'espèces indicatrices. Cela au niveau national alors que ce fructueux concept était, au travers des divers catalogues de types de stations, très régionalisé. Les auteurs de cet ouvrage nous indiquent d'ailleurs que ces groupes " permettent de réaliser un premier diagnostic stationnel en l'absence d'un catalogue des stations forestières; ils se révèlent être utilisables sur l'ensemble du territoire concerné [par chacun des deux volumes ${ }^{(2)}$ ]".

Ainsi, au-delà de la détermination des espèces, les objectifs de la Flore forestière sont, d'une part, d'aider à établir un diagnostic écologique simple (humidité/acidité), d'autre part, de participer à des études de relations entre végétation et facteurs du milieu ou d'en contrôler la cohérence. Ecoflore, le logiciel présenté ici, en informatisant cette double démarche, la rend plus aisée.

Pour tester la fiabilité d'un diagnostic à partir des informations de la Flore forestière, Bartoli et Largier (1992) avaient utilisé les groupes d'espèces indicatrices qu'elle propose, dans une étude des liaisons station-production pour le Merisier du piémont pyrénéen de la Haute-Garonne. Le niveau hydrique et le niveau trophique des stations y étaient mis en évidence à l'aide des "cocktails" des groupes propres à chaque station. Ayant obtenu un résultat positif très net, les auteurs notaient " qu'un logiciel capable de reproduire ces démarches permettrait un diagnostic rapide et de conserver en mémoire le détail des relevés pour une utilisation globale de l'information qu'ils contiennent».

Cette idée continuant à se discuter entre ses promoteurs, des élèves de la Formation d'Ingénieurs forestiers (Augé et al., 1993 ; Barot et Largier, 1993) avaient tenté des approches informatiques. Sous une autre forme, une maquette de logiciel utilisant une feuille de calcul Excel avait été créée (Bartoli, 1996). Un programme informatique plus convivial, développé par l'un d'entre nous - sous

(1) Flore forestière plus avant dans le texte.

(2) Plaines et collines d'une part, Montagnes de l'autre pour les deux tomes édités à ce jour. Le tome Région méditerranéenne est en cours de préparation. 
Visual Basic ${ }^{T M}$ et Access ${ }^{\top M}$ - et nommé Ecoflore est présenté ici. Les principes d'Ecoflore exposés, on en montrera la fiabilité, les limites et des exemples d'une première utilisation réalisée avec des relevés botaniques de l'Inventaire forestier national (IFN) et un développement informatique plus complet.

\section{LA STRUCTURATION DE LA FLORE EN GROUPES D'ESPÈCES INDICATRICES}

Les groupes d'espèces indicatrices correspondent à des " comportements moyens relativement superposables " (Rameau et al., op. cit.). La Flore forestière rattache chaque espèce végétale à un groupe d'espèces indicatrices en proposant une structuration en 31 groupes. Pour illustrer la valeur indicatrice de ces groupes et l'autécologie de chaque espèce, elle utilise toujours le même schéma de représentation : un diagramme niveau hydrique / niveau trophique - dit "écogramme" - qui donne une image, réduite mais claire, des situations écologiques relatives rencontrées vis-à-vis de ces deux facteurs primordiaux (figure 1, ci-dessous).

En effet, pour porter un diagnostic de fertilité correct, le croisement de données hydriques et de données trophiques semble suffisant. Tous les catalogues de types de stations font cette constatation. Dans la Flore forestière, certains des groupes d'espèces indicatrices intègrent de plus une autre donnée autécologique : la richesse du milieu en azote (espèces nitrophiles et nitroclines).

Ecoflore reprend ce classement proposé par la Flore forestière hormis pour un nombre réduit de cas - une vingtaine d'espèces sur plusieurs centaines décrites -, où nous avons été amenés à le modifier.

Ainsi Vaccinium myrtillus est passée du groupe des espèces acidiphiles de dysmoder et mor ( $A+)$ dans le groupe des espèces acidiphiles à large amplitude (A la). Cela est conforme à des obser-

Figure 1

À TITRE D'EXEMPLE, L'ÉCOGRAMME AVEC 4 (SUR 31) GROUPES D'ESPĖCES INDICATRICES DE LA FLORE FORESTIERRE

xx la : espèces xérophiles à large amplitude trophique

A+ : espèces acidiphiles de dysmoder et de mor

b : espèces calciclines

A la : espèces acidiphiles à large amplitude

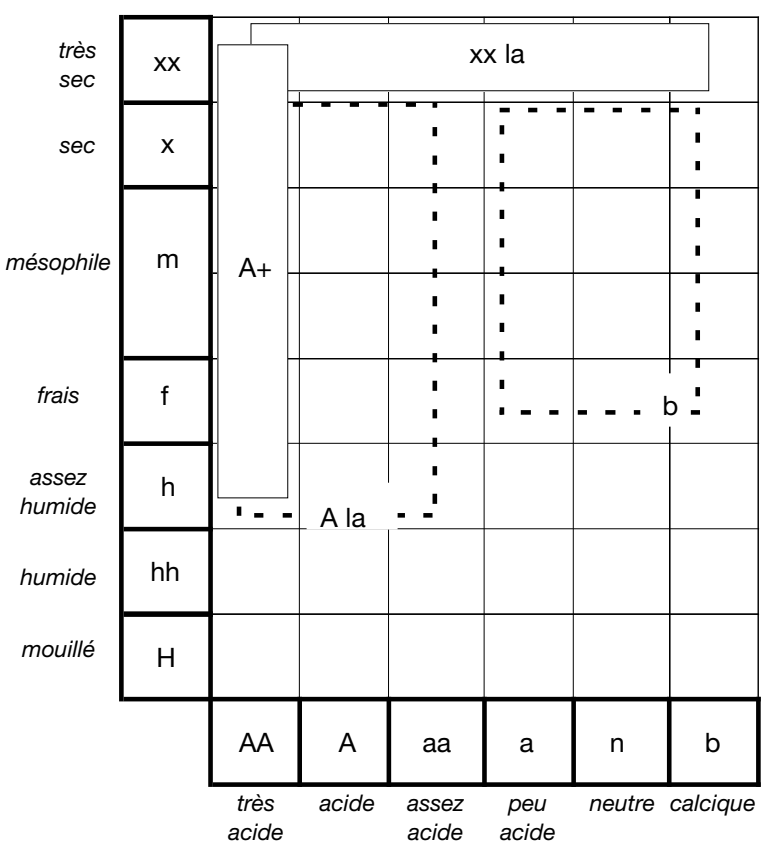




\section{BARTOLI - M. TRAN-HA - G. LARGIER - G. DUMÉ - L. LARRIEU}

vations comme celles de Richard (1961) (3) ou de Largier (1988) (3). De même, Hepatica nobilis, classée dans le groupe des xérophiles et mésoxérophiles calcaricoles et calcicoles, semble, au vu de son autécologie telle que décrite par la Flore forestière, mieux classée dans les neutrocalcicoles. L'appartenance du buis au groupe que lui assigne la Flore forestière a également été modifiée. Les raisons de ce dernier changement sont données plus loin car elles résultent de l'examen d'un test utilisant Ecoflore.

D'autres modifications, les plus nombreuses, sont justifiées par le fait que l'espèce est signalée deux fois (espèce dite bimodale) mais dans des groupes assez proches pour qu'ils soient rassemblés. Le cas des espèces perçues comme ayant une autécologie vraiment bimodale est traité à part. Enfin, nous avons séparé les calcaricoles des calcicoles à partir des indications de la Flore forestière.

\section{LE PRINCIPE DU LOGICIEL}

Nous avons développé notre programme en mettant nos pas dans ceux de la Flore forestière :

- il utilise l'écogramme qu'elle propose comme base de représentation,

- il retient les caractéristiques des groupes dans cette schématisation.

Chaque espèce est automatiquement rattachée à son groupe indicateur. Ecoflore calcule un barycentre. L'abscisse ( $\mathrm{Xr}$ : niveau trophique) et l'ordonnée ( $\mathrm{Yr}$ : niveau hydrique) du barycentre des espèces du relevé sont calculées en utilisant ${ }^{(4)}$ :

- l'abscisse ou l'ordonnée $G$ du centre du rectangle schématisant, dans l'écogramme, le groupe auquel appartient une espèce.

- le "poids interne" du groupe lié à la précision que les auteurs de la Flore forestière lui prêtent. Ce poids est différent suivant que l'on regarde le niveau trophique ou le niveau hydrique. Le groupe A+ (acidiphiles de dysmoder et de mor) apporte une forte information sur le plan trophique mais une faible information sur le plan hydrique. Pour tous les groupes, une pondération interne est ainsi prise en compte. Sur l'axe voulu, elle est l'inverse de la longueur relative $\left(\Delta_{\mathrm{i}}\right) \mathrm{du}$ côté du rectangle illustrant le groupe. Le groupe $A+$ a une largeur de 1 dans la coordonnée trophique de l'écogramme : son poids interne est de 1. Le groupe $A$ la, moins précis avec sa largeur de 2,5 sur l'axe des $x$ a donc un poids 2,5 fois plus faible $\left(1 / \Delta_{\mathrm{i}}\right)$.

- une correction $c$ de ce poids que nous justifions ci-après.

- un "poids externe" $(\mathrm{m})$ lié au coefficient d'abondance-dominance de l'espèce dans le relevé.

Nous avons utilisé directement le classique coefficient variant de + à 5 . À l'issue de quelques essais, "+" a été pris égal à 0,2.

À l'aide des relevés-types des types de stations d'un catalogue, comparant sur l'écogramme les situations de leurs barycentres, utilisant Ecoflore avec une pondération uniforme des groupes c'est-à-dire sans la correction "c" de leur poids interne - et celles que nous définissaient ces relevés, nous observions:

- un biais marqué pour le niveau hydrique : toutes les stations humides étaient estimées comme étant trop sèches et réciproquement ;

(3) Dans le Jura suisse, le premier ne considère " pas V. myrtillus comme une bonne espèce indicatrice. Bien qu'acidophile, elle est une véritable ubiquiste ". Pour une forêt des Pyrénées centrales, le second met en évidence une station à Hepatica nobilis et Vaccinium myrtillus tout en constatant, par ailleurs, l'existence, classique, d'une hêtraie acidiphile à myrtille.

(4) Pour i espèces, la formule complète est : $\operatorname{Xr}$ ou $\operatorname{Yr}=\left(\sum \mathrm{G}_{\mathrm{i}}{ }^{*} \frac{1}{\Delta_{\mathrm{i}}}{ }^{*} \mathrm{C}_{\mathrm{i}}{ }^{*} \mathrm{~m}_{\mathrm{i}}\right) /\left(\sum{\frac{1}{\Delta_{\mathrm{i}}}}^{*} \mathrm{c}_{\mathrm{i}}{ }^{*} \mathrm{~m}_{\mathrm{i}}\right)$. 
- un biais, plus faible mais dissymétrique, pour le niveau trophique : si les niveaux acides étaient à peu près correctement appréciés, les situations calcaricoles et calcicoles étaient décalées vers le pôle acide.

En pondérant le poids des groupes en fonction de leur position dans l'écogramme, ce biais s'atténue fortement alors que la forme de la représentation initiale de l'écogramme demeure. Cette pondération constitue la correction "c" qui intervient dans le calcul du barycentre.

La représentation ci-dessous montre que, dans la zone centrale - en blanc dans la figure $2-$, un certain "flou" dans le diagnostic est à attendre. Mais il affecte des conditions variant entre mésophile frais et mésophile sec et/ou peu acide et assez acide dont on comprendra qu'il est parfois difficile, pour le phytoécologue et pour... Ecoflore, d'en apprécier toutes les nuances.

Figure 2 PAR LE JEU DE PONDÉRATIONS DES GROUPES D'ESPĖCES INDICATRICES QUI SE SUPERPOSENT AUX DIVERSES ZONES PLUS OU MOINS FONCÉES,

\section{IL EST POSSIBLE DE RÉDUIRE LES BIAIS QU'UN ÉCOGRAMME NON CORRIGÉ FAIT APPARAîTRE}

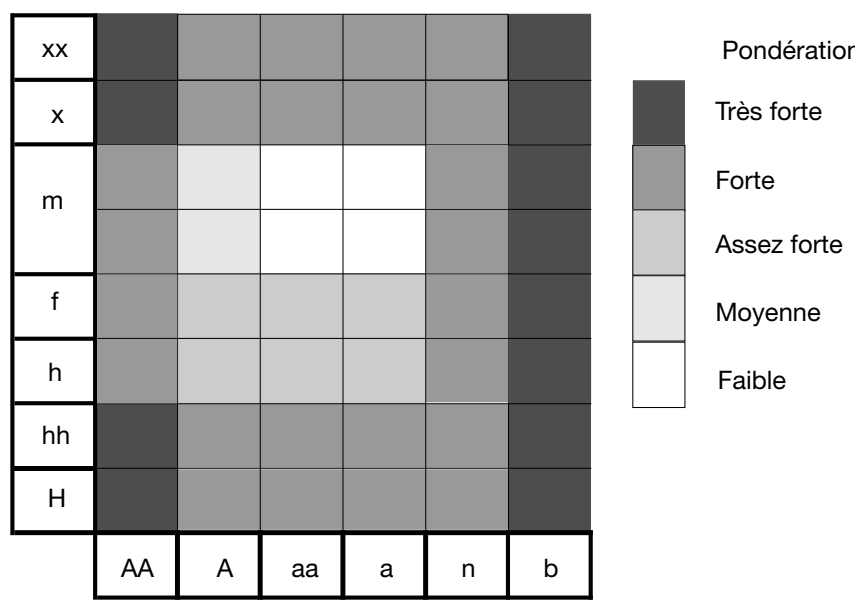

\section{L'UTILISATION D'ECOFLORE ET LES DOCUMENTS FOURNIS}

L'utilisateur peut intervenir directement sur tous les paramètres d'Ecoflore ${ }^{(5)}$ malgré les inconvénients d'un programme ainsi "ouvert". Les relevés sont enregistrables et pourront être réutilisés sous Ecoflore mais également exportables, notamment sous tout tableur ou autre gestionnaire de données, pour être rapprochés d'autres éléments écologiques ou cartographiques. Après une traduction informatique, il est possible d'importer un fichier de relevés déjà saisis sous un autre format.

Comme pour toutes les espèces, la Flore forestière fournit une image de l'autécologie des essences forestières. Sur le modèle de la schématisation des groupes d'espèces indicatrices, il était simple

(5) Paramètres de la formule de calcul du barycentre mais aussi rattachement des espèces à un groupe, création de groupes, introduction d'espèces nouvelles, de noms synonymes usités par l'utilisateur, etc. 


\section{BARTOLI - M. TRAN-HA - G. LARGIER - G. DUMÉ - L. LARRIEU}

de créer une représentation de ces enveloppes autécologiques pour les superposer à l'écogramme où figure le barycentre du relevé floristique d'une station. La base de données d'Ecoflore fournit, en standard, ces représentations pour les principales essences.

Les états imprimés fournis sont, comme l'illustre l'annexe (pp. 545-546) :

- le relevé floristique. Si on le désire, il sera classé dans l'ordre des groupes d'espèces indicatrices et comportera les coordonnées du barycentre ;

- un écogramme renseigné - identique à celui de la copie d'écran montrée - avec, au moins, le barycentre du relevé et, par exemple, les essences dont on veut visualiser l'intérêt compte tenu du diagnostic fait.

En utilisant le même principe que pour les essences, il est, de plus, possible de faire représenter sur l'écogramme d'Ecoflore :

- un type de stations. Sans doute est-il plus sûr et plus logique d'utiliser les clés des catalogues de stations locaux, mais on peut vouloir vérifier que la flore est bien un bon indicateur ou faire apparaître une éventuelle divergence de diagnostic.

- un type d'habitat, si tant est que l'on puisse le réduire à une représentation dans un écogramme.

\section{UN EXEMPLE DE RÉSULTAT : LES PLATEAUX CALCAIRES DE LORRAINE}

Les catalogues de types de stations forestières utilisant la flore comme bio-indicateur, fournissent, par type, au moins un relevé à titre d'exemple. Pour chacun des relevés-types, nous avons comparé la position du barycentre calculé par Ecoflore avec la représentation du type de station définie à partir des données du catalogue. Le schéma de droite de la figure 3 rassemble les positions relatives de tous les types: par exemple, les barycentres des types 2 et 14 sont décalés d'une seule case vers le bas (ils sont estimés trop humides par Ecoflore) par rapport à ce qui était attendu.

UTILISATION DES RELEVÉS-TYPES

\section{DU CATALOGUE DES TYPES DE STATIONS DES PLATEAUX CALCAIRES DE LORRAINE}

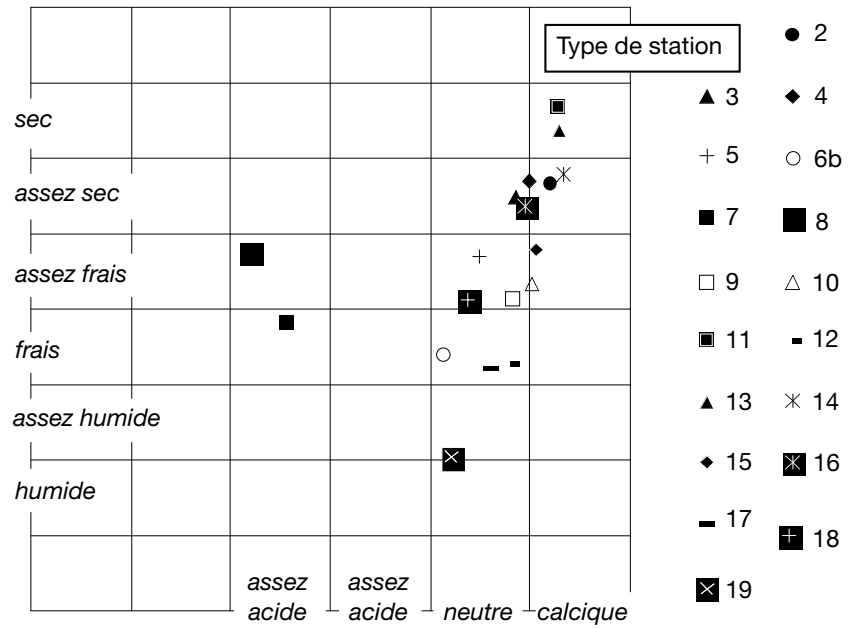

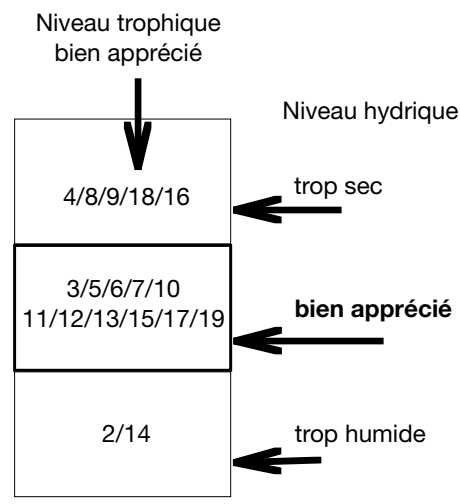


Le catalogue des types de stations des plateaux calcaires de Lorraine (Becker et al., 1980) a servi - et sert encore - de modèle aux catalogues français. S'il n'utilise pas d'écogramme pour situer les types les uns par rapport aux autres dans cette schématisation, les renseignements qu'il fournit sur le niveau trophique et le niveau hydrique associés à chaque type de stations permettent de situer chaque type avec précision dans ce schéma. La figure 3 montre les positions obtenues par Ecoflore à l'aide des relevés-types et les compare aux positions attendues.

Les numéros sont ceux des types de stations de ce catalogue.

Ainsi, pour une gamme stationnelle variée, l'image donnée par Ecoflore diffère fort peu de celle attendue et seul le diagnostic pour le niveau hydrique n'est pas très précis. Tous les tests, réalisés à partir de catalogues allant de l'étage subalpin de Haute-Maurienne (Bartoli, 1966) aux coteaux très atlantiques du Bas-Adour (Ackermann, 1994), en passant par le massif pré-alpin des Bauges (Boissier, 1996), les Pyrénées centrales (Savoie, 1995) ou le Perche (Charnet, 1988), ont été très satisfaisants.

\section{D'AUTRES UTILISATIONS D'ECOFLORE}

Avec, sans doute, un enthousiasme un peu prématuré, nous pensons qu'Ecoflore peut être utilisé non seulement pour effectuer des diagnostics écologiques corrects, mais aussi pour réaliser de nombreux travaux de phytoécologie.

\section{Mise en évidence d'une imprécision de la Flore forestière et d'une erreur de détermination botanique}

- Quel est le groupe d'espèces indicatrices du Buis ?

Dans le catalogue des Pyrénées centrales (Savoie, 1995), chaque type de stations est défini à partir d'une vingtaine de relevés. Nous avons utilisé les relevés du type mésotrophe frais de l'étage montagnard. Ce type - banal - comporte un grand nombre d'espèces variées (39 en moyenne, 185 au total). Nous avons représenté (figure 4) la dispersion des barycentres calculés pour tous les relevés de ce type de stations.

Figure 4

DISPERSION DES BARYCENTRES DES RELEVÉS DES STATIONS FORMANT LE TYPE MÉSOTROPHE FRAIS DU CATALOGUE DES PYRÉNÉES CENTRALES

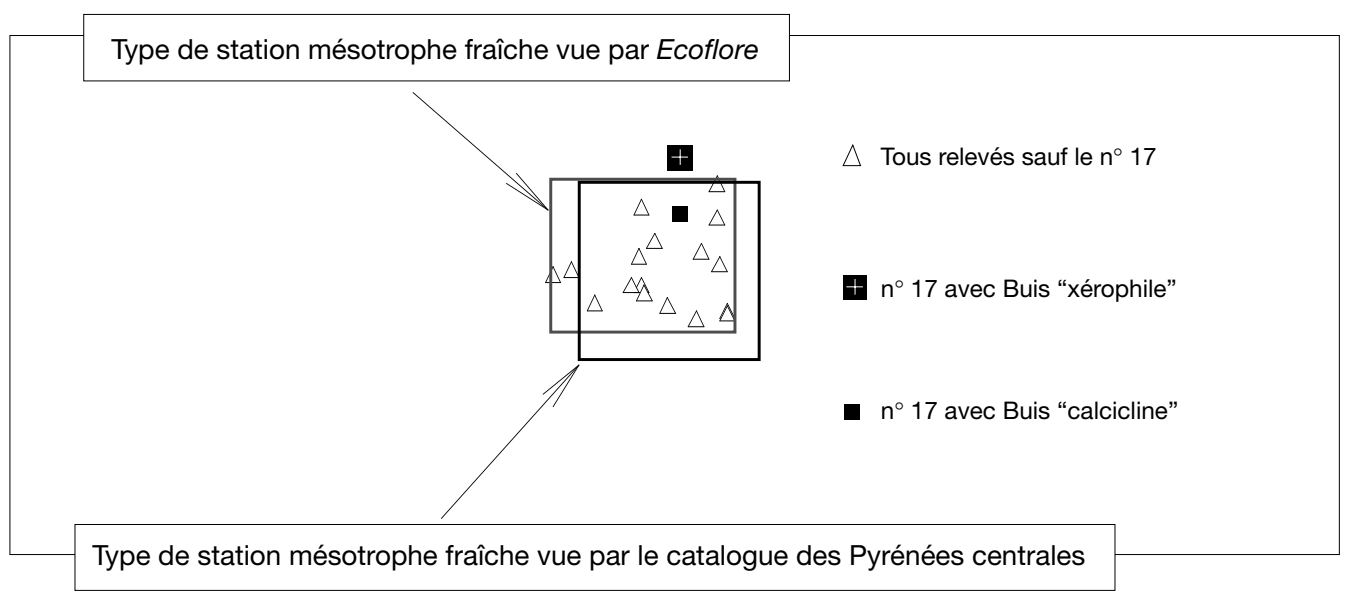




\section{BARTOLI - M. TRAN-HA - G. LARGIER - G. DUMÉ - L. LARRIEU}

La figure 4 (p. 535) permet de constater :

- la bonne précision générale fournie par Ecoflore par rapport à la situation schématisée dans l'écogramme du catalogue ;

- que l'indispensable homogénéité des relevés du type de stations visé est très forte dans ce cas ;

- la mauvaise position, toute relative, du relevé $n^{\circ} 17$, apprécié comme étant trop sec.

Ecoflore permet de voir, à l'écran, les raisons de la place du barycentre. Il est alors facile de constater que, pour ce relevé 17, c'est la seule présence du Buis qui explique l'éventuelle anomalie détectée. Le Buis est classé dans le groupe des "xérophiles à large amplitude trophique" (xx la) par la Flore forestière. La place de ce groupe dans l'écogramme lui confère un facteur de correction c élevé (voir § "le principe du logiciel", p. 532).

Figure 5

L'AUTÉCOLOGIE DU BUIS VUE PAR LA FLORE FORESTIĖRE COMPARÉE AU GROUPE DES XÉROPHILES À LARGE AMPLITUDE TROPHIQUE ET À CELUI DES CALCICLINES (se reporter à la figure 2, p.533)

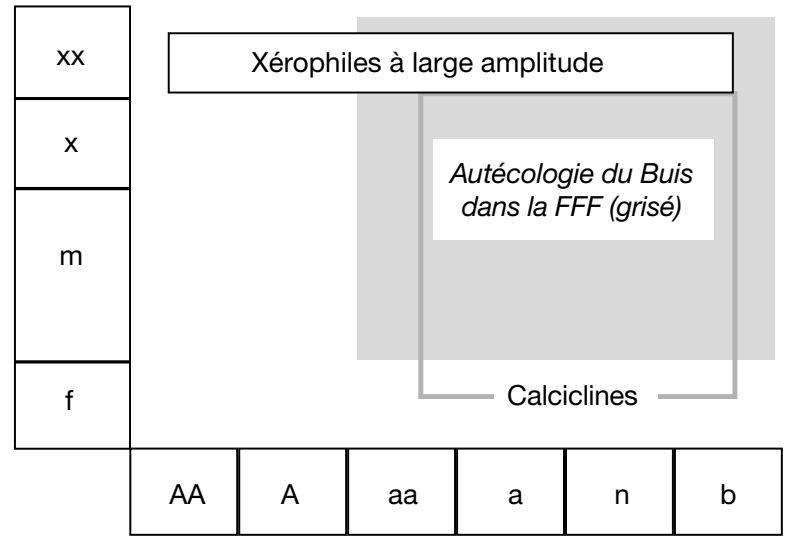

La figure 5 (ci-dessus) montre l'écart entre :

- d'une part, la représentation du groupe d'espèces des xérophiles à large amplitude (xx la) ;

- d'autre part, les données autécologiques propres au Buis fournies par la Flore forestière (zone en grisé). Le Buis est une espèce calcicline au sens de la Flore forestière et l'on peut dire que c'est par erreur qu'il est classé dans les espèces xérophiles.

Au moins dans les Pyrénées et les Préalpes du Nord, le Buis accompagne en effet, souvent de façon spectaculaire, les forêts neutrocalcicoles fraîches. Une fois le Buis affecté au groupe des calciclines dans Ecoflore, le résultat du calcul est immédiat. Le relevé 17 rejoint alors le groupe attendu (figure 4, p. 535).

- Confusion entre une espèce acidiphile et une espèce neutrocalcicole qui se ressemblent

Sur chaque placette de l'Inventaire forestier national (IFN), un relevé botanique aussi complet que possible est désormais réalisé (IFN, 1994). Il est associé à un relevé pédologique. Après avoir mis au point une chaîne de calcul automatique selon le principe d'Ecoflore, nous avons traité 202 relevés réalisés dans les régions "Front pyrénéen" et "Haute chaîne" des Hautes-Pyrénées (IFN, 1999). 
Pour contrôler la cohérence interne d'un relevé, un test de stabilité a été mis au point (6). II permet un tri entre les espèces qui laissent le diagnostic à peu près stable et celles qui le déplacent. $\mathrm{Ce}$ test, automatisé, consiste à recalculer le barycentre en retirant, tour à tour, une des espèces constitutives du relevé. Le test permet de repérer l'espèce (ou les espèces) qui génère un écart important, au moins une demi-"unité" sur l'un des axes de l'écogramme pour notre exemple.

84 relevés, soit $42 \%$ du total, sont concernés par cette importante instabilité. Si l'on observe quelles sont les espèces causes de ces variations, nous en trouvons 38 pour le seul niveau trophique. Le tableau ci-dessous en présente les principales : celles qui apparaissent dans au moins 10 relevés :

\begin{tabular}{|c|c|c|c|}
\hline $\begin{array}{l}\text { Espèce entraînant la plus forte variation } \\
\text { du niveau trophique calculé }\end{array}$ & $\begin{array}{c}\text { Nombre de relevés } \\
\text { comportant l'espèce } \\
\text { (sur 202) }\end{array}$ & $\begin{array}{l}\text { Nombre de relevés } \\
\text { concernés }\end{array}$ & $\%$ \\
\hline Deschampsia flexuosa . . . . . . . . & 102 & 46 & 45 \\
\hline Lonicera xylosteum & 10 & 2 & 20 \\
\hline Helleborus foetidus & 17 & 3 & 18 \\
\hline Rhododendron ferrugineum & 15 & 2 & 13 \\
\hline Daphne laureola . . . . . . . . . . & 47 & 6 & 13 \\
\hline
\end{tabular}

Considérons le cas de la Canche flexueuse (Deschampsia flexuosa) qui fait partie du groupe d'espèces indicatrices des acidiphiles de moder mésophiles. Lorsqu'on retire cette espèce des 46 relevés fortement perturbés par cet enlèvement, les barycentres glissent tous nettement vers un niveau moins oligotrophe. La figure 6 (p. 538) illustre les situations des 46 relevés dont le barycentre s'est sensiblement déplacé par retrait de la Canche flexueuse.

Trois hypothèses peuvent expliquer ce phénomène :

- dans des relevés de niveau trophique oligotrophe - où le nombre d'espèces est très faible et où la Canche a une forte abondance -, nous supprimons une caractéristique essentielle du niveau trophique ;

- dans les Pyrénées, cette espèce n'a pas la même autécologie qu'ailleurs ;

- la Canche est confondue avec une espèce qui lui ressemble et qui est d'autécologie différente. Dans les Pyrénées, la Canche flexueuse a pu être confondue, hors période de floraison, avec la Fétuque de Gautier (Festuca gautieri $=F$. scoparia), endémique pyrénéo-ibérique fréquente - mais méconnue dans les hêtraies sur calcaire - et neutrocalcicole.

La figure 6 (p. 538) permet de repérer correctement les 15 à 20 cas qui relèvent certainement de I'hypothèse 3 , ceux dont le barycentre initial se situe aux niveaux peu oligotrophe et assez oligotrophe et qui passe au niveau neutre sans la "fausse Canche". Seul un retour terrain permettra de lever l'incertitude apparue.

Lors des deux exemples qui précèdent, nous n'avons pas cherché à prouver qu'Ecoflore fournit de meilleurs diagnostics que ceux réalisés par les techniques habituelles, mais qu'il peut être un filtre efficace pour mettre en évidence une partie des incohérences qui peuvent apparaître dans un ensemble de relevés.

(6) II est dû à E. Bruno (IFN de Montpellier). 
M. BARTOLI - M. TRAN-HA - G. LARGIER - G. DUMÉ - L. LARRIEU

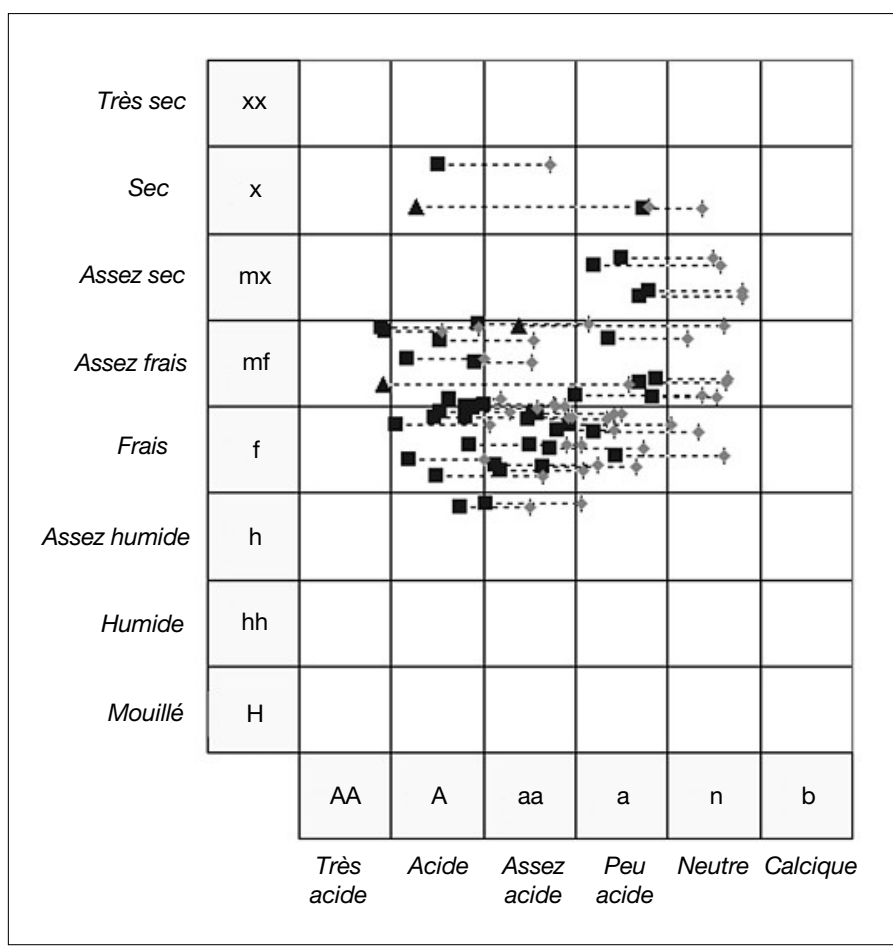

Figure 6

DÉPLACEMENT DU BARYCENTRE

CALCULÉ PAR ECOFLORE

DES 46 RELEVÉS OÙ LE RETRAIT

DE LA CANCHE FLEXUEUSE

PROVOQUE

UNE FORTE VARIATION

DE LA POSITION DU BARYCENTRE

Carrés noirs $=$ avec la Canche

Losanges gris = sans la Canche

Les trois triangles correspondent aux points qui se déplacent le plus

\section{Relativiser les écogrammes dans les catalogues de types de stations}

Tester la fiabilité d'Ecoflore est simple lorsque des écogrammes schématisent le niveau hydrique et le niveau trophique des types de stations. Cela est le cas dans les catalogues de types de stations les plus récents. Le plus souvent, l'écogramme de la Flore forestière paraît être utilisé comme référence absolue. C'est, par exemple et pour le niveau hydrique, le cas du catalogue des Pyrénées centrales déjà cité. Dans des situations de pentes toujours bien drainées, le niveau "assez humide" du catalogue ne correspond, pour l'indice d'Ecoflore, qu'au niveau "frais" de l'écogramme de la Flore forestière. Ce décalage montre - en fait - une appréciation relative de ces niveaux suivant les situations générales de la région étudiée.

De même, le niveau mésotrophe du catalogue du Vercors (en cours d'élaboration) ne sera certainement pas le même que celui qualifié de mésotrophe dans le contexte, globalement plus humide, de la Chartreuse (Pache, 1998). Ainsi, en fournissant une valeur quantifiable de "valeur nationale" pour les différents types de divers catalogues, Ecoflore peut permettre de réaliser des passerelles entre leurs niveaux hydriques et trophiques.

\section{Précision de diagnostics issus de relevés plus ou moins complets}

Ecoflore peut permettre de répondre rapidement à des questions relatives à la précision écologique d'un diagnostic issu d'un relevé plus ou moins complet :

- L'estimation de l'indice d'abondance-dominance est sujette à un fort effet-observateur. Un relevé en simple présence/absence permet-il un diagnostic proche de celui tenant compte de cet indice? 
- Quelle peut être l'influence de la non-prise en compte des bryophytes sur la précision de notre diagnostic ? Ce cas est assez fréquent :

- des difficultés de détermination peuvent entraîner des biais a priori gênants ;

- il est parfois difficile de se rendre compte si la mousse est une véritable humicole ou si elle n'est pas posée sur un résidu de souche ou autre caillou. Dans ces cas, sans utiliser des éléments d'autécologie trompeurs, les espèces auront à apparaître dans le relevé mais ne devront pas être prises en compte dans le calcul du barycentre.

- De même, il est évident que les espèces peu fréquentes sur les placettes de relevés risquent d'échapper à l'observateur. Le constat fait lors des contrôles réalisés sur le réseau RÉNÉCOFOR (7) (Dobremez et al., 1997) montre que ces oublis peuvent être assez importants. Nous avons fait un test, sans doute trop sévère, qui écarte des calculs toutes les espèces dont le coefficient d'abondance-dominance n'est que "+".

Ce sont à nouveau les 19 relevés du type "mésotrophe frais" des Pyrénées centrales qui ont été utilisés pour comparer ces quatre situations. Le " 0 " correspond au relevé complet prenant en compte les coefficients d'abondance-dominance. L'écart - 0,5/ + 0,5 est celui de la largeur d'une case de l'écogramme, c'est-à-dire la dimension unitaire souvent donnée à la représentation d'un type de station. Nous présentons le résultat pour l'estimation du seul niveau hydrique :

Figure 7

VARIATION DU DIAGNOSTIC EN CAS DE NON-PRISE EN COMPTE DU COEFFICIENT D'ABONDANCE-DOMINANCE (A/D), c'est-à-dire en simple présence/absence, en cas de non-utilisation des bryophytes ou en cas de non-prise en compte des espèces rares dans le relevé lui-même

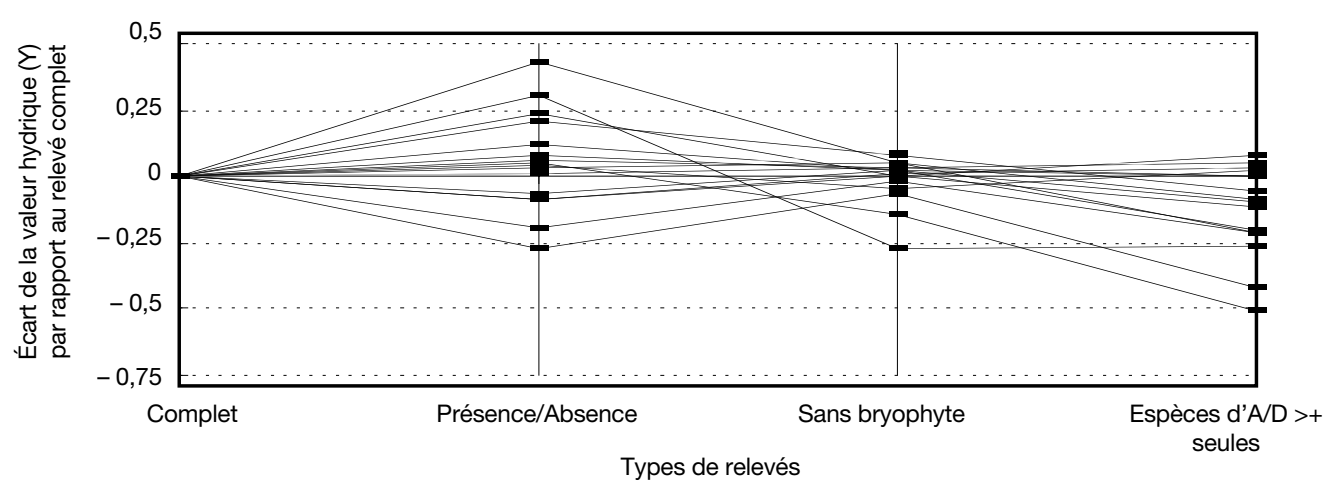

Il faut se garder de généraliser ce qui est issu de très peu de relevés ayant, par leur appartenance à un même type de stations, des structures et des espèces similaires, mais :

- ne pas tenir compte de l'abondance fait assez fortement diverger le diagnostic, cela de façon à peu près symétrique ;

- si les bryophytes sont écartées de l'analyse, le diagnostic reste très correct ;

- écarter toutes les espèces peu fréquentes montre bien que toutes les espèces ont un rôle à jouer dans l'interprétation des caractéristiques du milieu. Dans la réalité d'un relevé floristique, toutes les espèces d'abondance-dominance "+" ne sont pas ainsi oubliées comme nous l'avons fait ici.

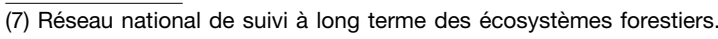




\section{BARTOLI - M. TRAN-HA - G. LARGIER - G. DUMÉ - L. LARRIEU}

\section{Comparaison avec des indices d'Ellenberg}

Ce n'est pas la première fois que les valeurs indicatrices quantitatives sont comparées à des valeurs qualitatives de facteurs de l'environnement. Suivant cette approche, les travaux les plus connus sont ceux d'Ellenberg et al. (1992) qui analysent la flore de l'Allemagne. Des valeurs de 1 à 9 définissent l'optimum de chaque espèce pour six indices dont deux ("humidité du sol pendant la saison de végétation" et "acidité du sol") sont, de toute évidence, tout à fait comparables, respectivement, à l'abscisse et l'ordonnée du barycentre calculées par Ecoflore.

Lors de la mise en place du réseau RÉNÉCOFOR, la composition floristique de 101 peuplements a permis le calcul des indices d'Ellenberg ${ }^{(8)}$. Comparant les indices synthétiques d'Ellenberg calculés et une information concernant les facteurs pédologiques (la forme d'humus), nous avons noté des discordances entre cette donnée écologique et l'indice de niveau trophique. Elles nous ont paru assez nombreuses pour que nous envisagions de réaliser une comparaison entre les valeurs indicatrices d'Ellenberg et celles d'Ecoflore pour ce facteur. Nous estimons que la forme d'humus a été correctement évaluée.

\begin{tabular}{|c|c|c|c|c|c|}
\hline $\begin{array}{l}\mathrm{N}^{\circ} \text { des placettes } \\
\text { RENECOFOR }\end{array}$ & Forme d'humus & $\begin{array}{l}\text { Classe par } \\
\text { les indices } \\
\text { d'Ellenberg }\end{array}$ & $\begin{array}{c}\text { Appréciation } \\
\text { sur l'estimation } \\
\text { fournie par l'indice } \\
\text { d'Ellenberg jugée }\end{array}$ & $\begin{array}{c}\text { Valeurs } \\
\text { indicatrices } \\
\text { d'Ecoflore }\end{array}$ & Commentaire \\
\hline & & Sol & & Sol & \\
\hline CHS 27 & Moder & acide à peu acide & trop peu acide & acide & $\begin{array}{l}\text { acidité bien appréciée } \\
\text { par Ecoflore }\end{array}$ \\
\hline CHS 57a & Mésomull & neutre & idem & assez acide & $\begin{array}{l}\text { acidité bien appréciée } \\
\text { par Ecoflore }\end{array}$ \\
\hline CHS 60 & Oligomull & peu acide à neutre & idem & peu acide & $\begin{array}{l}\text { acidité bien appréciée } \\
\text { par Ecoflore }\end{array}$ \\
\hline DOU 23 & Moder - Hémimoder & acide à peu acide & idem & acide & $\begin{array}{l}\text { acidité bien appréciée } \\
\text { par Ecoflore }\end{array}$ \\
\hline HET 02 & Moder - Hémimoder & peu acide à neutre & idem & peu acide & $\begin{array}{l}\text { acidité mieux appréciée } \\
\text { par Ecoflore }\end{array}$ \\
\hline HET 30 & Dysmoder - Moder & acide à peu acide & idem & acide & $\begin{array}{l}\text { acidité bien appréciée } \\
\text { par Ecoflore }\end{array}$ \\
\hline HET 54a & Mésomull - Oligomull & peu acide à neutre & idem & assez acide & $\begin{array}{l}\text { acidité bien appréciée } \\
\text { par Ecoflore }\end{array}$ \\
\hline HET 76 & Hémimoder à moder & acide à peu acide & idem & acide & $\begin{array}{l}\text { acidité bien appréciée } \\
\text { par Ecoflore }\end{array}$ \\
\hline
\end{tabular}

L'indice trophique d'Ecoflore est systématiquement en meilleur accord avec le type d'humus que ne l'est l'indice d'Ellenberg. Sous réserve de réaliser d'autres essais, nous pensons que les indices d'Ecoflore pourraient être utilisés pour la France entière. De même, ces indices peuvent certainement être des moyens objectifs de comparaison de modifications de milieux comme celles mises en évidence, grâce à la Flore, sur le long terme par Dupouey et al. (1998) ou, sur le court terme, d'une perturbation liée à une exploitation forestière (Deconchat, 1999).

(8) Valeur des indices d'Ellenberg pondérée par le recouvrement de chaque espèce. Chaque valeur fait partie d'une classe : sol très sec, sol très acide... 


\section{Utilisation lors de l'élaboration d'un catalogue de type de stations}

Réaliser un diagnostic écologique à l'aide de la Flore exige - ce que confirme Ecoflore - de fortes et complètes connaissances en botanique et un bon "coup d'œil écologique" de la part de l'observateur qui doit, en tant qu'expert, vérifier que les autres descripteurs sont bien en cohérence avec celui-là. Ecoflore ne permet donc pas d'établir un catalogue des stations. Un type de stations ne se réduit pas à la seule végétation et il ne saurait être question d'utiliser seulement Ecoflore pour rapprocher des groupes de relevés. De plus, sauf pour quelques cas alors signalés, Ecoflore n'appréhende pas les éventuels écotypes régionaux. Des travaux sur l'autécologie des espèces, comme ceux de Gégout et Pierrat (1998), pourront permettre d'améliorer la précision de cet outil.

Inversement, un catalogue classe les espèces dans des groupes socio-écologiques en faisant abstraction de situations non représentées dans sa région étudiée. Si des groupes ainsi définis sont pertinents à l'échelle de cette région, les différences de comportement entre espèces sont plus clairement reflétées dans un territoire plus vaste comme l'ont montré les travaux de Brisse et al. à partir de la banque de données phytosociologiques Sophy (voir Ruffray et al., 1989). Le fait qu'Ecoflore fournisse des résultats corrects pour des régions de France variées et contrastées va dans le même sens.

II nous semble qu'Ecoflore peut apporter des éléments de réflexion au moment où s'élabore un catalogue. En particulier :

- Les types de stations que permet de mettre en évidence une analyse statistique au sein d'un ensemble de relevés lors de l'élaboration d'un catalogue sont choisis parmi les stations de la région. Ecoflore permet d'avoir un regard plus global en fournissant une autécologie nationale moyenne. Une comparaison critique entre les deux résultats peut permettre à une personne très expérimentée de juger ce qui ressort d'une précision autécologique régionale d'une simple appréciation statistique.

- À condition d'utiliser la base de données constituée à partir des groupes écologiques régionaux issus du catalogue, Ecoflore paraît pouvoir aider à choisir les relevés qui illustreront le type de stations et sa variabilité.

- Utilisant une méthode tout à fait indépendante de celle, statistique, habituellement utilisée, Ecoflore peut permettre de comparer les deux résultats. II doit alors être possible de détecter rapidement des divergences de diagnostic et de mieux positionner, dans l'écogramme, les types des stations mis en évidence.

\section{Une appréciation des habitats forestiers d'une région}

Dans les Pyrénées centro-occidentales, suivant les travaux des phytosociologues, la description des hêtraies est déclinée suivant une combinaison du gradient trophique et du gradient hydrique. Cette déclinaison, basée principalement sur la Flore, a été reprise par la nomenclature CORINE Biotopes pour définir des types d'habitats. À partir du travail de rapprochement entre ces types d'habitats et les types de stations forestières des Pyrénées centrales réalisé par Savoie (1996), il est possible de représenter les types d'habitats sur un écogramme même si, on le conçoit, il s'agit là d'une vision passablement réductrice de ce qu'est un habitat.

Une fois calculées ses coordonnées à l'aide d'Ecoflore, un relevé va donc pouvoir être rapproché, en première approximation, d'un habitat forestier. À partir des données de I'Inventaire forestier national (IFN, 1999) (9), il est possible d'obtenir une idée quantitative, en surface, des habitats des hêtraies de cette zone des Hautes-Pyrénées comme l'illustre la figure 8 (p. 542).

(9) Nous remercions I'IFN d'avoir bien voulu nous permettre l'accès à ses riches données écologiques. 
M. BARTOLI - M. TRAN-HA - G. LARGIER - G. DUMÉ - L. LARRIEU

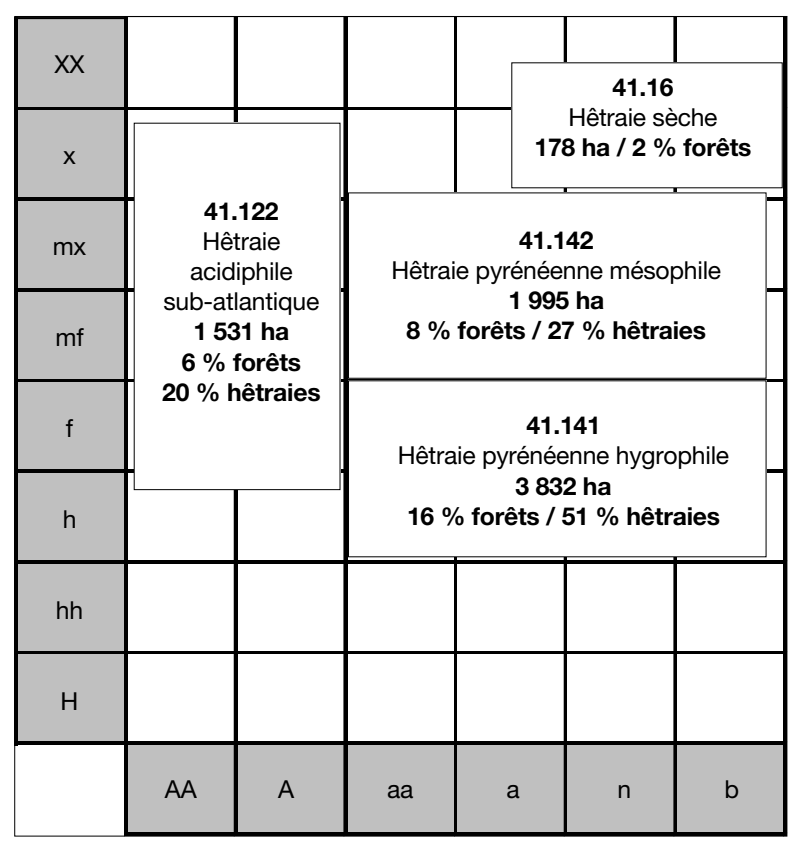

Figure 8

RÉPARTITION DES HABITATS DES HÊTRAIES

DANS LA ZONE ÉTUDIÉE

DES PYRÉNÉES CENTRALES

$\left(n^{\circ}=\right.$ code CORINE)

Notons que la richesse hydrique et trophique mise en évidence (51\% des hêtraies sont de type hygrophile) est a priori remarquable quant à leur productivité. Mais il ne faut pas perdre de vue qu'une exploitabilité souvent délicate ou que des traitements antérieurs, futaie sur souche ou sylviculture trop "timide" (Chollet et Demarcq, 1998), ne lui permettent pas toujours de s'exprimer économiquement.

Ces éléments, compatibles avec un nouveau langage européen, celui de CORINE Biotopes, viendront enrichir les renseignements que fournissent les données de l'IFN. Mais si elle est possible pour les hêtraies des Pyrénées occidentales, bien stratifiées, elle sera à appréhender par d'autres moyens dans la plupart des cas, le découpage de CORINE n'étant pas toujours une hiérarchisation du croisement niveau hydrique / niveau trophique.

\section{Un outil pédagogique}

Dans le format sous lequel il sera diffusé dans un premier temps, Ecoflore ne traite qu'un relevé à la fois. Sous cette forme, il a déjà, à notre avis, un très net intérêt pédagogique. Essayé par une classe de Techniciens supérieurs option gestion forestière, il a permis (Rimasson, in litt.) « de faire comprendre le passage de la notion d'autécologie d'une espèce végétale à l'indication apportée par un ensemble d'espèces (relevés) et un ensemble de relevés ».

\section{CONCLUSIONS}

Ecoflore utilise la Flore forestière, ouvrage accessible déjà bien connu des enseignants, des élèves et des praticiens forestiers français ou voisins. En conséquence, bien entendu, le passage par les groupes d'espèces indicatrices - et non directement par les données autécologiques propres à chacune des espèces - est le fondement du logiciel. La précision et la fiabilité des résultats 
obtenus montrent que la Flore forestière est bien, selon la formule de Jacamon (1990), un « bel outil de travail ».

Nous voyons Ecoflore comme un instrument écologique supplémentaire dans le laboratoire d'analyses de terrain de tous les forestiers et enseignants qui, connaissant très correctement la botanique, se promènent déjà avec un $\mathrm{pH}$-mètre et une tarière pédologique et utilisent ces outils pour réaliser un diagnostic stationnel. Précisons qu'Ecoflore est déjà prêt à recevoir les informations d'autécologie du futur tome "Méditerranée" de la Flore forestière.

Ecoflore a les limites de tout outil utilisant des indicateurs écologiques : la cohérence du diagnostic doit être vérifiée par l'examen d'autres descripteurs du très complexe écosystème forestier. Nous espérons qu'il sera aussi vu comme un reflet de la capacité, toujours fascinante, du monde végétal à s'adapter et à interagir avec le milieu.
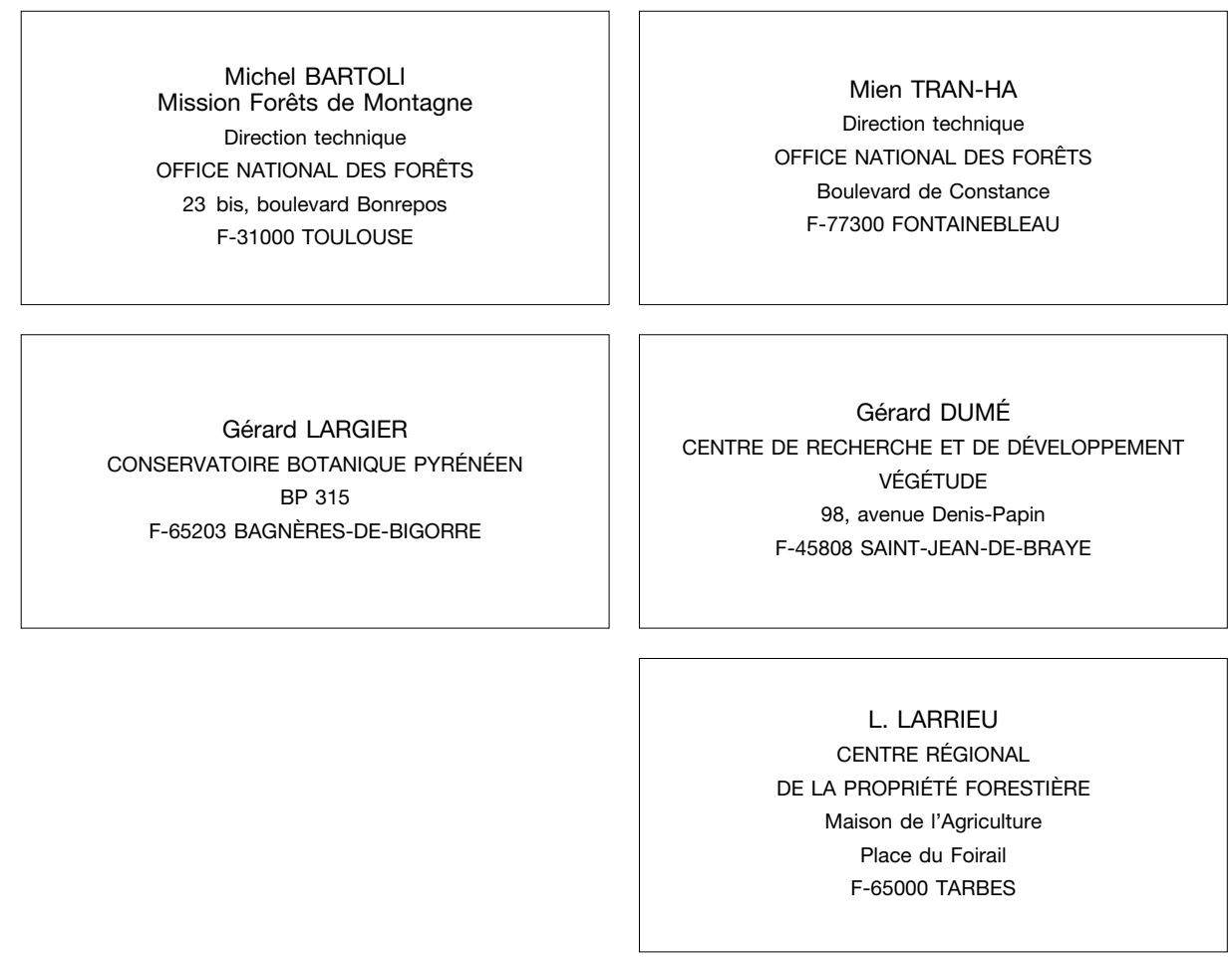

La mise au point d'Ecoflore a bénéficié du soutien financier de l'Office national des Forêts au titre des "études innovantes".

Au prix de $150 \mathrm{~F}$ TTC, Ecoflore est disponible auprès de M. Bartoli. Préciser le support souhaité : CD ou disquettes (PC seulement). La diffusion interne à l'ONF sera assurée ultérieurement. 


\section{BARTOLI - M. TRAN-HA - G. LARGIER - G. DUMÉ - L. LARRIEU}

\section{BIBLIOGRAPHIE}

ACKERMANN (F.). - Catalogue des types des stations forestières de la Chalosse, du Bas-Adour et des coteaux basques et béarnais. - CEMAGREF, 1994. - $506 \mathrm{p}$.

AUGÉ (V.), MARSOL (L.), RIOND (C.), ROMANSKI (M.). - Création d'un logiciel de diagnostic stationnel à partir de la Flore forestière française. - Projet informatique de la FIF-ENGREF, 1993. - $14 \mathrm{p}$.

BAROT (S.), LARGIER (G.). - Diagnostic stationnel (niveau trophique et niveau hydrique) à partir de la démarche proposée par la Flore forestière française (Rameau et al., 1988). - Projet informatique de la FIF-ENGREF, 1993. - $14 \mathrm{p}$.

BARTOLI (Ch.). - Études écologiques sur les associations forestières de Haute-Maurienne. - Annales des Sciences forestières, tome XXIII, fascicule 3, 1966, 321 p. (Thèse).

BARTOLI (M.). - Le Logiciel Isopyrénées : flore et diagnostic stationnel. - Document interne de la "Rencontre technique d'Aspet : autour des stations forestières des Pyrénées centrales". - ONF, 1996. - 6 p.

BARTOLI (M.), LARGIER (G.). - Utilisation pratique de la Flore forestière française pour réaliser un diagnostic écologique. - Bulletin technique de l'ONF, n²3, 1992, pp. 55-72.

BECKER (M.), LE TACON (F.), TIMBAL (J.). - Les Plateaux calcaires de Lorraine. Types de station et potentialités forestières. - Nancy : ENGREF, 1980. - $216 p$.

BOISSIER (J.-M.). - Le Massif des Bauges. Types de stations et relations station-production. - Université Joseph-Fourier, Grenoble I, 1996. - 172 p.

CHARNET (F.). - Catalogue des types de station forestière du Perche (Eure-et-Loir, Loir-et-Cher). - CRPF de I'lle de France et du Centre, 1988. - 583 p.

CHOLLET (F.), DEMARCQ (P.). - Réaction des hêtraies de montagne aux éclaircies tardives. - Revue forestière française, vol. L, n 4, 1998, pp. 349-356.

DECONCHAT (M.). - Exploitation forestière et biodiversité ; exemple dans les forêts fragmentées des coteaux de Gascogne. - Université de Toulouse III, 1999. - 185 p. (Thèse).

DOBREMEZ (J.-F.), CAMARET (S.), BOURJOT (L.), ULRICH (E.), BRÊTHES (A.), COQUILLARD (P.), DUMÉ (G.), DUPOUEY (J.-L.), FORGEARD (F.), GAUBERVILLE (C.), GUEUGNOT (J.), PICARD (J.-F.), SAVOIE (J.-M.), SCHMITT (A.), TOUFFET (J.), TREMOLIËRES (M.). - RENECOFOR - Inventaire et interprétation de la composition floristique de 101 peuplements du réseau (campagne 1994/95). - Fontainebleau : Office national des Forêts - Département des Recherches techniques, 1997. - 513 p.

DUPOUEY (J.-L.), BECKER (M.), BERT (D.), CADEL (G.), LEFÈVRE (Y.), PICARD (J.-F.), THIMONIER (A.). Évolution récente des sols, de la végétation et de la productivité des forêts de montagnes françaises. Écologie, vol. 29, $\mathrm{n}^{\circ} 1-2,1998, \mathrm{pp} .341-349$.

ELLENBERG (H.), WEBER (H.E.), DÜLL (R.), WIRTH (V.), WERNER (W.), PAULIßEN (D.). - Zeigewerte von Pflanzen in Mitteleuropa. - Scripta Geobotanica, 18, 1992, pp. 1-258.

GÉGOUT (J.-C.), PIERRAT (J.-C.). - L’Autécologie des espèces végétales : une approche par régression non paramétrique. - Écologie, vol. 29, n³, 1998, pp. 473-482.

IFN. - $3^{e}$ inventaire du département des Hautes-Pyrénées. - Inventaire forestier national, 1999.

JACAMON (M.). - La Flore forestière française : un précieux document scientifique. Un bel outil de travail. Revue forestière française, vol. XLII, $\mathrm{n}^{\circ}$ 5, 1990, pp. 513-515.

LARGIER (G.). - Approche stationnelle d'une hêtraie montagnarde, la forêt domaniale de Cagire (HauteGaronne). - Office national des Forêts STIR Sud-Ouest - Certificat de typologie des stations forestières, 1988. - $68 \mathrm{p}$.

PACHE (G.). - Catalogue détaillé des stations forestières du massif de la Chartreuse et des chaînons calcaires du Pays "Entre Jura-Savoie". - Université Joseph-Fourier, Grenoble I, 1998. - 306 p.

RAMEAU (J.-C.), MANSION (D.), DUMÉ (G.). - Flore forestière française. Tome 1 : plaines et collines. - Paris : IDF, 1989. - $1784 \mathrm{p}$.

RAMEAU (J.-C.), MANSION (D.), DUMÉ (G.). - Flore forestière française. Tome 2 : montagnes. - Paris : IDF, 1992. $-2421 \mathrm{p}$.

RICHARD (J.-L.). - Les Forêts acidiphiles du Jura, étude phytosociologique et écologique. - Berne : Éd. Huber, 1961. - $164 \mathrm{p}$.

RUFFRAY (P. de), BRISSE (H.), GRANDJOUAN (G.), HOFF (M.). - "Sophy", une banque de données phytosociologiques. In : Les plantes sauvages menacées de France, Actes du Colloque de Brest. - BRG, 1989. pp. $129-150$.

SAVOIE (J.-M.). - Les Types de stations forestières des Pyrénées centrales. - Toulouse : ONF Midi-Pyrénées, 1995. $-507 \mathrm{p}$. 


\section{ANNEXE}

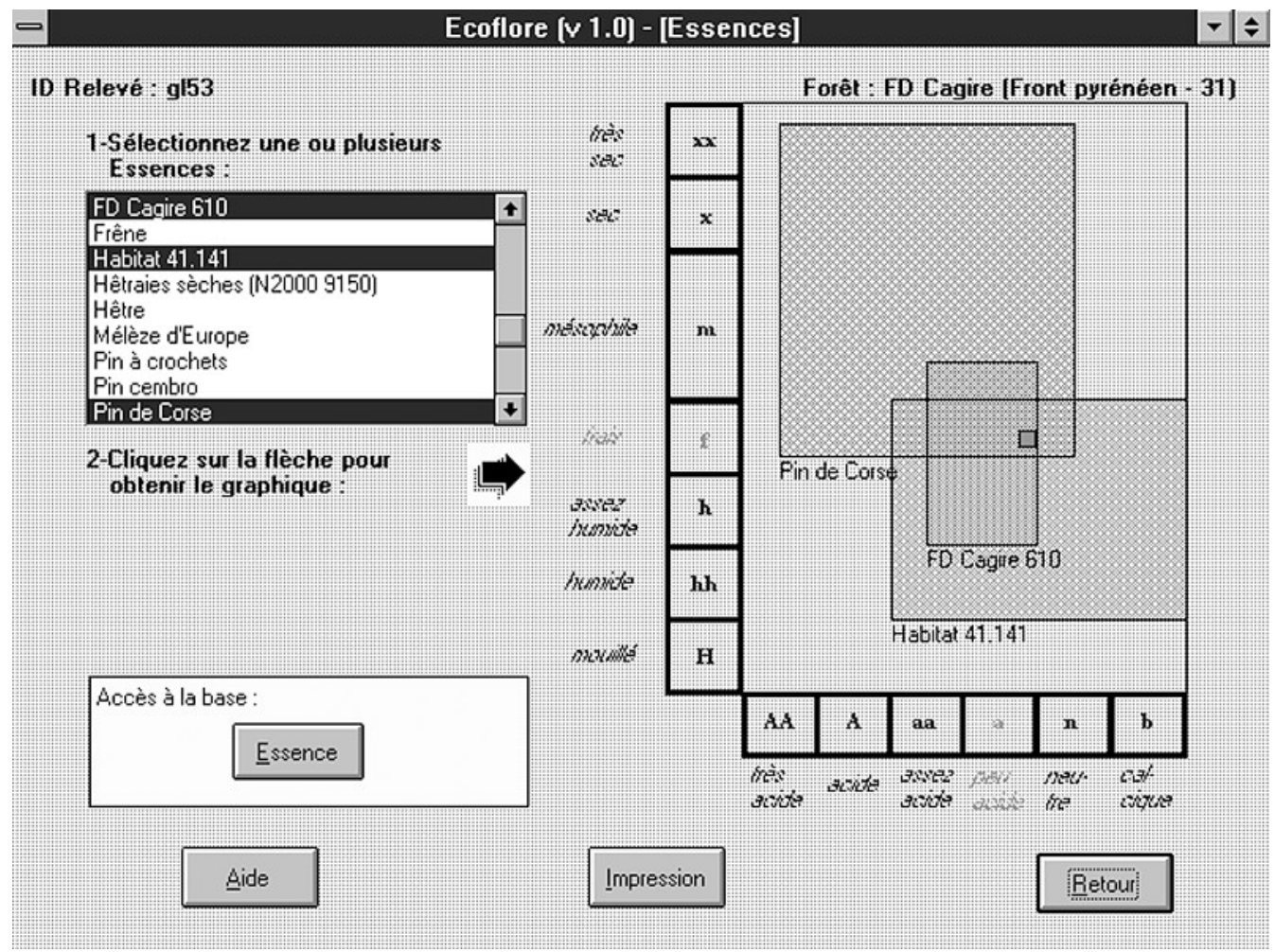

Copie de l'un des écrans d'Ecoflore

Sur l'écogramme présenté à l'écran, une fois réalisée la sélection (fonds noirs) sur le menu déroulant de gauche, apparaissent les enveloppes schématisant :

- le type de station 610 du catalogue de Largier (1988) ;

- I'habitat des Hêtraies pyrénéennes hygrophiles (Scillo-Fagetum p.) codé 41.141 par la nomenclature CORINE Biotopes ;

- l'autécologie du Pin laricio de Corse telle que présentée par la Flore forestière.

Cette dernière information est contenue dans les bases de données fournies avec le logiciel.

Les deux précédentes sont à saisir par l'utilisateur dans le module dit "GEl".

Le barycentre (petit carré noir) qui se positionne automatiquement correspond, ici, au relevé $\mathrm{n}^{\circ} 53$ réalisé lors de la mise au point du catalogue de Largier (1988) pour une forêt des Pyrénées centrales. II fournit un diagnostic conforme à celui attendu. En effet, ce relevé avait été rattaché par l'auteur au type de station 610 (Hêtraie-sapinière acidicline fraîche à Athyrium filix-femina).

Ecoflore imprime ce relevé en classant les espèces dans les groupes d'espèces indicatrices de la Flore forestière (voir p. 546). Les coordonnées du barycentre sont indiquées. 
M. BARTOLI - M. TRAN-HA - G. LARGIER - G. DUMÉ - L. LARRIEU

Relevé : $g 153$

Forêt : FD de Cagire (Front pyrénéen - 31)

Relevé type du type 610 (acidicline frais)

GEI : Acidiclines de mull mésotrophe hygroclines

Athyrium filix-femina

Fougère femelle

Veronica montana

Véronique des montagnes

1 ATHF ah

GEI : Acidiphiles à large amplitude

Blechnum spicant

Luzula sylvatica
Blechne en épi

Luzule des bois
+ VERM a h

+ BLES A la

1 LUZS A la

GEI : Acidiclines de mull mésotrophe mésophiles

Epilobium montanum

Rubus groupe fruticosus

Dryopteris affinis $2 \mathrm{ssp}$.

Dryopteris affinis $2 \mathrm{ssp}$.
Epilobe des montagnes

Ronce des bois

Dryoptéris voisin

Dryoptéris voisin

Oxalide petite oseille

Cardamine à sept folioles
+ EPIM a m

1 RUBX a m

+ DRYA a m

+ DRYA a m

\section{GEI : Hygrosciaphiles}

Oxalis acetosella
Cardamine heptaphylla

1 OXAA HsC

+ CARH Hsc

\section{GEI : Neutroclines à large et moyenne amplitudes}

Corylus avellana

Hedera helix

Hypericum androsaemum

Lamiastrum galeobdolon

Melica uniflora

Fissidens taxifolius

Polystichum setiferum

Pulmonaria affinis

\section{GEI : Neutrocalcicoles}

Lathyrus laevigatus

GEI : Neutronitroclines hygroclines

Fraxinus excelsior a

Cardamine pratensis

GEI : Neutronitroclines mésophiles

Hypericum hirsutum

Noisetier

Lierre

Androsème

Lamier galeobdolon

Mélique uniflore

Fissident à feuilles d'if

Polystic à cils raides

Pulmonaire semblable

Gesse jaune

Frêne commun a

Cardamine des prés

Millepertuis velu

Scrofulaire alpestre

Ortie dioïque

\section{Scrophularia alpestris}

Urtica dioica

GEI : Espèces à très large amplitude
Fagus sylvatica
Abies alba
Quercus petraea g

\section{GEI non déterminé}
Carex montana*

(28 espèces)
Hêtre

Sapin pectiné

Chêne sessile (semis)

Laîche des montagnes* $\begin{array}{lll}3 & \text { CORA } n \text { am et } n \text { la } \\ 1 & \text { HEDH } n \text { am et } n \text { la } \\ + & \text { HYPA } n \text { am et } n \text { la } \\ + & \text { LAMG } n \text { am et } n \text { la } \\ 1 & \text { MELU } n \text { am et } n \text { la } \\ 1 & \text { mFIT } n \text { am et } n \text { la } \\ 2 & \text { POSE } n \text { am et } n \text { la } \\ + & \text { PULA } n \text { am et } n \text { la }\end{array}$

1 LATL nB

+ FRWE nN h

+ CAPR nN h

$1 \mathrm{HYPH} n \mathrm{n} \mathrm{m}$

+ SCRA nnNNh

+ URTD nnNNh

4 FAGS tla

+ ABIA tla

+ QUZP tla

+ CAM1 nB ou n la

Coordonnées du relevé dans l'Ecogramme: $x=3.82 \quad y=3.41$ 


\section{ECOFLORE, UN LOGICIEL SIMPLE DE DIAGNOSTIC ÉCOLOGIQUE (Résumé)}

La structuration en groupes d'espèces indicatrices et l'utilisation systématique d'un diagramme humidité/acidité pour schématiser l'autécologie des espèces végétales présentées par la Flore forestière française (Rameau et al., 1989 et 1992) ont permis de développer un logiciel, nommé Ecoflore. À partir d'un relevé de la végétation, il calcule un barycentre dans les coordonnées hydriques et trophiques du diagramme. Testée en comparant l'emplacement de ce barycentre et ce qui était une situation attendue au travers de nombreux relevés-types de divers catalogues de types de stations forestières, sa précision se révèle très bonne. Il a été utilisé pour mettre en évidence des déterminations incorrectes d'espèces, pour fournir des diagnostics écologiques dans des zones sans catalogue, pour juger de l'imprécision que l'on peut avoir si l'on utilise ou pas les bryophytes ou les coefficients d'abondance-dominance. II permet de choisir des essences dont l'autécologie est connue et est un excellent outil pédagogique.

\section{ECOFLORE, A SIMPLE SOFTWARE APPLICATION FOR AN ECOLOGICAL DIAGNOSIS (Abstract)}

A structure by groups of indicative species and the systematic use of a humidity/acidity diagram to plot the autecology of the plant species presented in Flore forestière française (Rameau et al., 1989 and 1992) were used to develop a software application named Ecoflore. Based on a vegetation map, it calculates a barycenter in the water and trophic co-ordinates of the diagram. When the position of the barycenter is tested against the situation as derived from numerous standard records in the various catalogues of forest site types, its accuracy is very good. It has been used to pinpoint the incorrect species determination, to provide ecological diagnoses in areas where catalogues are not available, to assess uncertainty depending on whether or not bryophyta or frequency/dominance coefficients are used. It can be used to select species whose autecology is known and is furthermore an excellent teaching aid. 